\title{
Literatura e historia. A propósito de las políticas de memoria desde las bibliotecas escolares de Río Gallegos
}

\section{Literature and history. Memory policies since the school libraries of Río Gallegos}

\author{
Ana Cejas, Pablo Navas \\ cejas.anita.gisela@gmail.com, pablonavasrg@gmail.com \\ Unidad Académica Río Gallegos - Universidad Nacional de la Patagonia Austral \\ Piloto Rivera s/n - Río Gallegos - Santa Cruz - Argentina
}

Recibido: 01/07/2021. Aceptado: 22/10/2021

\begin{abstract}
RESUMEN
El presente trabajo propone un abordaje que examina el rol que les cabe a las escuelas de la provincia de Santa Cruz en el proceso de construcción de la(s) memoria(s) colectiva(s) particularmente de dos hechos traumáticos de la historia contemporánea santacruceña: por un lado, las huelgas de los peones rurales de 1920/21 y, por el otro, la última dictadura cívicomilitar de 1976. Si bien ambos hechos contienen su propia densidad histórica y particularidades propias, lo cierto es que ambos hechos han irrumpido en el calendario de actos escolares. Por eso nos interesa analizar los mecanismos que se activan en relación al recuerdo/olvido, y de qué modo se implementan no sólo en los días señalados por la efeméride escolar, sino también en la cotidianeidad más extensa de las instituciones escolares. Desde esta perspectiva, nos interesa retomar el análisis respecto al lugar de las bibliotecas escolares en la construcción de la memoria colectiva santacruceña.
\end{abstract}

Palabras clave: Memoria colectiva; bibliotecas escolares; Santa Cruz.

\begin{abstract}
This work proposes an approach that examines the role that the schools of the province of Santa Cruz play in the process of construction of the collective memory(s), particularly of two traumatic events in contemporary Santa Cruz history: on the one hand, the rural peon strikes of 1920/21 and, on the other, the last civic-military dictatorship of 1976. Although both events contain their own historical density and peculiarities, the truth is that both events have disrupted the calendar of school events. That is why we are interested in analyzing the mechanisms that are activated in relation to remembering/forgetting, and in what way they are implemented not only on the days indicated by the school anniversary, but also in the more extensive daily life of school institutions. From this perspective, we are interested in resuming the analysis regarding the place of school libraries in the construction of the collective memory of Santa Cruz.
\end{abstract}

Keywords: Collective memory; School libraries; Santa Cruz. 


\section{INTRODUCCIÓN}

Uno de los principios con los que quizás más se insiste a quienes se aproximan a la disciplina de la historia es que el pasado resulta constantemente reelaborado según las sensibilidades éticas, culturales y políticas del presente. La tarea de los/as historiadores/as, nada sencilla por cierto, es identificar y ubicar su producción científica en ese devenir o tránsito.

El abordaje del pasado desde la ciencia histórica es sólo una instancia del proceso de producción social de la memoria sobre el pasado que desarrollan las sociedades. Memoria e historia lejos de ser sinónimos se constituyen como dimensiones con ambiciones diferentes. La memoria es sostenida por la sociedad en su conjunto, por eso, se encuentra en evolución permanente, abierta a la dialéctica del recuerdo y de la amnesia inconsciente a sus deformaciones sucesivas, vulnerable a todas las utilizaciones, manipulaciones, susceptible de pasar por largas etapas de latencia o súbitas revitalizaciones. Por otro lado, la Historia es la reconstrucción siempre problemática e incompleta de lo que no está más. La historia en tanto operación intelectual y secular, reclama análisis y discurso crítico. La memoria instala el recuerdo en lo sagrado, la historia lo extrae de allí y lo hace prosaico (Pierre Nora: 2009; 20-21).

$\mathrm{La} / \mathrm{s}$ memoria/s se van construyendo a partir de productos culturales muy heterogéneos: investigaciones científicas, testimonios en una sala de tribunal, archivos privados y álbumes de fotos de familia e incluyendo el propio currículum escolar. De esta manera, alcanzan un nivel de institucionalización a partir del ordenamiento en los museos, transformado en espectáculo, ritualizado, reificado, el recuerdo del pasado. De esta manera se transforma en memoria colectiva una vez que ha sido seleccionado y reinterpretado según las sensibilidades culturales, los interrogantes éticos y las conveniencias políticas del presente.

Como advertimos más arriba, las preguntas por la memoria producen diferentes movimientos en el plano de lo social por tratarse de una textura laxa que selecciona entre los escombros del pasado.

La historia de la provincia de Santa Cruz está atravesada por dos hechos traumáticos, el primero de ellos fue el proceso de represión estatal y civil a huelguistas rurales desarrollada entre los años 1920-1921, conocido popularmente como la Patagonia Rebelde. El segundo hecho, más cercano en el tiempo, está vinculado a la acción represiva llevada adelante en el marco de la última dictadura cívico-militar en la Argentina entre 1976-1982. En último caso, de acuerdo a las investigaciones de la Secretaría de Estado de Derechos Humanos de la provincia de Santa Cruz, se registraron 13 desapariciones forzadas. ${ }^{1}$ Está claro que ambos procesos históricos dolorosos presentan contornos de evocación con sus propias particularidades. En relación a la dictadura en Santa Cruz, durante algunas décadas estuvo instalada la imagen de que en la provincia patagónica el accionar terrorista del Proceso de Reorganización Nacional, no había pasado más allá de algunas cesantías de empleados públicos provinciales, y de que había un único desaparecido, el diputado provincial Juan Carlos Rosell.

\footnotetext{
${ }^{1}$ De acuerdo a las averiguaciones del Estado provincial a través de la Secretaría de Estado de Derechos Humanos, uno de los casos de detención tuvo lugar en la capital santacruceña, Río Gallegos, y el resto de detenciones y/o de desapariciones forzadas de personas tuvieron lugar en las ciudades de Buenos Aires, La Plata y Córdoba. Se trata de los casos de Margarita Delgado, Reinaldo Rampoldi, Ricardo Alberto Cittadini, Pedro Llorente Serrano, Oscar Walmir Montoya, Delmiro Segundo Villagra, Inés Magdalena Uhalde, Juan Carlos Rosell, Viviana Admetlla, Daniel Alberto Toninetti, Héctor Manuel Irastorza, Andrés Armendáriz Leache y Adriana Cecilia Barcia.
} 
Sobre la represión a los huelguistas rurales de la Patagonia Rebelde, en el centenario de los hechos, también se puede realizar una trazabilidad de evocación identificando sus pliegues heterogéneos. Durante largas décadas, los hechos represivos hacia los peones rurales fueron insinuados bajo la perspectiva de las clases dominantes locales, caracterizándose por una valoración positiva del accionar del ejército y el desmedido (antipatriótico) reclamo de los trabajadores urbanos y rurales, que ponían en peligro estos territorios. Como complemento de esta perspectiva hegemónica se estableció un silencio sobre los fusilamientos y las persecuciones. Salvo escasas excepciones, esto se sostuvo hasta el retorno a la democracia. A partir de 1983 con la amplia difusión del libro y la película de Osvaldo Bayer, "La Patagonia Rebelde", emergieron una serie de "otras memorias" sobre las huelgas que habían quedado en el ámbito de lo privado. Muchas de estas se originaron a partir de la acción de organizaciones sociales que rescataron la acción de los huelguistas y visibilizaron la represión cívico-estatal en el entonces Territorio Nacional de Santa Cruz. Estas acciones fueron impulsando procesos de revisión que pusieron en cuestionamiento las interpretaciones hegemónicas. En tiempos más recientes, el Estado de la Provincia de Santa Cruz, ha iniciado una política activa de memoria sobre estos hechos históricos.

Proponemos entonces realizar un acercamiento inicial a los procesos de construcción de la memoria oficial de esos dos acontecimientos traumáticos en la histórica contemporánea de la provincia de Santa Cruz, haciendo foco en las bibliotecas de las instituciones educativas.

\subsection{Resultados análisis y discusión (Metodología)}

\section{La función de las bibliotecas escolares según la legislación}

Hemos advertido al inicio sobre las complejidades en el vínculo entre memoria e historia, nuestro análisis insiste en revisar el rol que le cabe a las escuelas en el proceso de construcción de la memoria colectiva: cómo opera, qué mecanismos de recuerdo u olvido utiliza, de qué modos lo implementa. Seguramente, podamos responder a esa pregunta evocando las diferentes actividades llevadas a cabo por parte de los docentes en fechas como el 24 de Marzo, o a nivel provincial -muy recientemente- en vistas del 7 de Diciembre con la conmemoración de las Huelgas Patagónicas acontecidas en territorio santacruceño ${ }^{2}$.

Sin embargo, más allá de las fechas conmemorativas, corresponde la reflexión sobre cómo esos diferentes procesos históricos se traducen en la cotidianeidad de las escuelas. Es por ello que en este trabajo nos interesa retomar el lugar de las bibliotecas escolares en la construcción de la memoria colectiva.

Los hechos históricos mencionados produjeron agrietamientos difíciles de saldar colocando a las instituciones educativas en un territorio de formulación de interrogantes que no sólo convoquen a revisar el pasado, sino que también permitan encontrar nuevos sentidos al devenir del presente. La construcción de una "pedagogía de la memoria" implica reflexionar sobre algunas cuestiones vinculadas a problemas que se desprenden de la propia tarea de la transmisión y que tienen que ver fundamentalmente con las preguntas por los contenidos y las formas en las que se enseña ese pasado y cómo hacerlo.

\footnotetext{
${ }^{2}$ Feriado Provincial Permanente el día 7 de diciembre de cada año, en conmemoración a los obreros fallecidos en las denominadas "Huelgas Patagónicas" sucedidas en territorio santacruceño LEY 3.668 RIO GALLEGOS, 26 de septiembre de 2019 Boletín Oficial, 8 de Octubre de 2019 Vigente, de alcance general Id SAIJ: LPZ0003668
} 
Es importante señalar que el resultado de la indagación de este trabajo se ha visto condicionada por las restricciones que el Estado provincial fue adoptando en función de la adhesión a diversas normas dictadas por el Poder Ejecutivo Nacional en materia de emergencia sanitaria vinculada al COVID-19, que componen el "Distanciamiento Social, Preventivo y Obligatorio". En este sentido las instituciones escolares se han mantenido funcionando en la virtualidad y restringiendo toda actividad presencial. Esto último nos ha impedido el acceso a las instituciones escolares para realizar el relevamiento en las propias bibliotecas escolares.

Producto de este condicionante, un punto de partida del relevamiento fue revisar las menciones que se realizan sobre las bibliotecas escolares dentro de los colegios, en la legislación vigente en materia de educación en los derechos humanos.

La memoria y los Derechos Humanos son contenidos obligatorios de algunas asignaturas escolares. Así lo establece la Ley Nacional de Educación $N^{\circ} 26.206$ que en su artículo 92 propone incluir estas temáticas en los contenidos de todas las jurisdicciones con el objetivo de generar sentimientos democráticos y de defensa del Estado de Derecho y la plena vigencia de los Derechos Humanos.

Complementariamente la Ley de Educación Nacional prevé dotar "a todas las escuelas de los recursos materiales necesarios para garantizar una educación de calidad, tales como la infraestructura, los equipamientos científicos y tecnológicos, de educación física y deportiva, bibliotecas y otros materiales pedagógicos, priorizando aquéllas que atienden a alumnos/as en situaciones sociales más desfavorecidas, conforme a lo establecido en los artículos 79 a 83 de la presente ley" (Ley de Educación Nacional, 2006, Artículo 87). En otro de los artículos de la mencionada Ley también se establece un acuerdo entre el Ministerio de Educación, Ciencia y Tecnología y el Consejo Federal de Educación que tiene como objetivo fortalecer "las bibliotecas escolares existentes y asegurará su creación y adecuado funcionamiento en aquellos establecimientos que carezcan de las mismas". Asimismo, implementará planes y programas permanentes de promoción del libro y la lectura" (Ley de Educación Nacional, 2006, Artículo 91). Por su parte, la provincia de Santa Cruz adhirió a la mencionada ley nacional a través de la sanción de la Ley de Educación Provincial N³305 en el año 2012. La misma no suma elementos nuevos sobre las bibliotecas escolares a la jurisdicción provincial ya que replica lo citado en su artículo 141 inciso e) y artículo 147 respectivamente.

En la línea de la normativa vigente también se puede mencionar la Ley Nacional № 26.917 correspondiente al Sistema Nacional de Bibliotecas Escolares y Unidades de Información Educativas, sancionada en el 2013 y promulgada en el 2014. Esta ley se enmarca dentro de la Ley de Educación Nacional mencionada más arriba y si bien profundiza sobre los fines del Sistema Nacional propone algunos objetivos de interés para este trabajo como ser "promover líneas de acción institucionales orientadas a favorecer la lectura crítica y reflexiva en las unidades de información de cada comunidad educativa, como modo de comprensión de su realidad pasada y presente a nivel individual, social y cultural, en coordinación con los planes de lectura nacionales y jurisdiccionales" (Ley Nacional Sistema Nacional de Bibliotecas Escolares y Unidades de Información Educativas, 2013, Artículo 5).

En relación a lo planteado en torno a los planes de lectura nacionales es oportuno mencionar que a través de ellos diversos libros que fueron censurados dentro del último período de dictadura cívico-militar en Argentina fueron recuperados y colocados a disposición de forma digital en la página oficial del Plan Nacional de Lectura (http://planlectura.educ.ar/). El listado de aquellos libros censurados puede encontrarse en el anexo del trabajo de investigación $L a$ 
literatura infantil argentina, de los años '60 y '70 al presente: resistencia y continuidad literaria (Pac y Cejas, 2020, pp. 220-221). *Es de interés para esta investigación poder realizar un trabajo de campo en diferentes bibliotecas escolares de la ciudad para profundizar sobre la presencia de estos libros en formato papel y la utilización de los mismos en las aulas.

\section{Otra mirada sobre las bibliotecas escolares: funciones y el rol del bibliotecario}

A propósito de las funciones atribuidas a las bibliotecas escolares, Michèle Petit (2011) nos invita a reflexionar sobre el uso y la utilidad que tienen hoy en día estos espacios en la comunidad. Describe diversas experiencias en torno a bibliotecas en diferentes lugares del mundo -de interés resulta la mención a una actividad desarrollada en la localidad de Las Herasy destaca la importancia de la lectura, el acercamiento a estos espacios que, en contextos complejos, permite reconocer las bibliotecas como un lugar de resguardo y reflexión sobre la realidad, o creación de una realidad posible. Por ello escribe en un pasaje, en alusión a la lectura que "sirve no solamente para enunciar nuestra experiencia singular sino también para expandir los límites al infinito" (Pètit, 2011. p.5). En relación con los ejes de este trabajo -procesos de construcción de memoria colectiva- resulta oportuna la propuesta repensar la literatura, en sus diferentes formatos como "un soporte muy notable para despertar la interioridad, poner en movimiento el pensamiento, reanimar la actividad de simbolización y construcción de sentido, y también incita a experiencias de compartir inéditas" (Pètit, 2011. p.6). Así es que se busca, a lo largo del trabajo, revalorizar el espacio bibliotecario: no como un dispositivo didáctico más, sino como un espacio cultural, un conservatorio de sentido. Con ello no es nuestra intención desmerecer los esfuerzos de los diferentes organismos por lograr una óptima sistematización de las bibliotecas del país, pero sin dudas la lectura de estos trabajos (como de otros mencionados más adelante) nos permite atribuirle a la biblioteca otras funciones que no se encuentran por escrito.

En otro apartado, Pétit (2011) nos invita a pensar mediante testimonios a la biblioteca como un lugar humano dando lugar a revisar el rol del bibliotecario. Este último toma un gran protagonismo al momento de volcar la vista sobre las bibliotecas escolares, evocando a la vivencia individual es difícil imaginarse el paso por estos espacios sin el acompañamiento y calidez de los encargados de mantener todo en orden y proveer la información necesaria. Pero esto último resulta escueto si consideramos los diferentes trabajos escritos por los mismos bibliotecarios: "más allá de nuestras tareas técnicas de organización y difusión de la información que, si bien son fundamentales en nuestro quehacer diario, no deben ser un fin en sí mismo sino una herramienta (Bossié, 2009, p. 10). Florencia Bossié (2009) nos brinda una mirada en torno a la presencia, función y participación de los bibliotecarios en los diferentes procesos históricos. En este trabajo se refiere específicamente al lugar que ocuparon dentro de la última dictadura cívico-militar en Argentina y es de sumo interés las palabras finales que realiza a propósito de ello. Por un lado ,es oportuno mencionar la carga ideológica en la clasificación y las normas para el acceso, si se toma en cuenta que requiere de una gran responsabilidad aquel recorte que se realiza para colocar el material a disposición de los lectores. Adquiere aún más importancia el aspecto ideológico si consideramos que es mediante esas decisiones que se toman por parte de bibliotecarios que se delinea una forma de construir la memoria o la 'desmemoria' tal como menciona Bossié: "la memoria se construye también con olvidos y es cada persona en su subjetividad, recordando algunos hechos y desechando otros, la que consolida la memoria colectiva, de la cual es parte constitutiva también la desmemoria" (2009, p. 11). Puede decirse, entonces, que allí radica la importancia de las bibliotecas escolares en la construcción de la memoria colectiva. Más adelante Bossié agrega 
"qué son los libros sino objetos de memoria, qué son los documentos de archivos, sino huellas que nos permiten reconstruir una vida, unos actos, una identidad" $(2009$, p. 11) por lo que, podemos concluir, que el acceso a ellos con el acompañamiento de un bibliotecario a la altura de las circunstancias es fundamental a los ojos de este trabajo y los fines que persigue.

Para retomar la impronta de lo ideológico, Valdivia (2018) a propósito de las bibliotecas y la memoria menciona que una "opción es asumir que las bibliotecas también formamos parte en esta construcción y que las decisiones que tomamos en torno a las colecciones y a los servicios destinados a nuestros usuarios promueven ciertos hábitos -y no otros- respecto del flujo de información" (p.6). A lo largo de su escrito hace alusión a literatura censurada en el período dictatorial y considera la importancia de que aquellos libros sean recuperados y circulen en los contextos escolares. Sin embargo, reconoce que no es suficiente el hecho de que sean publicados y distribuidos en los colegios (podríamos considerar al Plan Nacional de Lectura), en consecuencia, escribe "bien sabemos que lo que no circula, no se lee, no se media, está destinado al olvido. $\mathrm{Y}$ ahí es donde entramos en juego los bibliotecarios. Por fuera de los circuitos meramente comerciales y aún de los circuitos oficiales, los caminos que se generen desde las bibliotecas y que promovamos quienes las habitamos, las prácticas de lectura que habilitemos o clausuremos son lo que mantendrá en movimiento esta construcción de memoria colectiva" (Valdivia, 2018, p. 7)

\section{Hacia la construcción de una pedagogía de la memoria traumática}

Es importante señalar que las acciones sobre políticas sobre la memoria dentro del ámbito educativo en la provincia de Santa Cruz, se desarrollaron de manera complementaria con el impulso registrado desde el gobierno nacional a los procesos de revisión del pasado reciente, de los que derivó el juzgamiento de los genocidas de la última dictadura argentina, beneficiados en muchos casos por las leyes de impunidad. ${ }^{3}$

Vale señalar que durante la segunda mitad de la década del noventa del siglo pasado, las luchas por la memoria en Argentina tomaron un nuevo impulso: apareció en escena la agrupación HIJOS (Hijos por la Justicia contra el Olvido y el Silencio) con nuevas prácticas - como los "escraches"- que acompañaban la ronda de las Madres de Plaza de Mayo. Esta emergencia fue acompañada por la aparición de otras voces: la de los militares "arrepentidos"4. En este contexto, caracterizado como de un "boom de la memoria" (Lvovich y Bisquert 2008; Cerruti 2001), comenzaron a aparecer detalles olvidados o silenciados en la construcción de la memoria, como la militancia política previa de muchos de los desaparecidos. Esta memoria reivindicativa de la militancia puso en discusión la idea de "víctimas inocentes" que había instalado el Nunca más y el Juicio a las Juntas (González 2014, 8).

\footnotetext{
${ }^{3}$ La cuestión de la memoria emerge en Argentina en relación con los crímenes, desapariciones, torturas y persecuciones perpetrados por el terrorismo de Estado impuesto por la última dictadura militar (Vezzetti 2002; Jelin 2002). Aunque las luchas por la memoria habían comenzado con la temprana labor de los organismos de derechos humanos durante la dictadura — por ejemplo, las Madres de Plaza de Mayo-, se puede afirmar que la construcción de la memoria se vincula fuertemente con la investigación llevada a cabo por la Comisión Nacional sobre la Desaparición de Personas (CONADEP), la publicación de sus resultados en el informe Nunca más y el Juicio a las Juntas.

${ }^{4}$ Uno de los ejemplos que se puede citar es el testimonio del marino Adolfo Scilingo quien confesara detalles de las metodologías para la desaparición de cuerpos, dando muestras de un plan sistemático de exterminio que desarmaba, al mismo tiempo, la narrativa de los "errores y excesos" de la "guerra sucia". A esta situación se sumó la autocrítica pública del general Balza, otrora jefe de las fuerzas armadas.
} 
Es en este escenario que el propio Estado Nacional, se involucró en el proceso de recuperación de las huelgas patagónicas de manera muy activa. Entre los ejemplos que pueden señalarse, fue la demarcación en el año 2010 con los pilares de memoria, verdad y justicia por parte de la Dirección Nacional de Sitios de Memoria, dependiente del Archivo Nacional de la Memoria (ANM), del cenotafio construido frente a la Estancia La Anita, cercana a la localidad de El Calafate, donde se conmemora todos los 8 de diciembre el fusilamiento de los obreros rurales por parte del ejército.

Esta acción resulta destacable puesto que el cenotafio de la estancia La Anita resultó el primer sitio en ser incluido dentro de los espacios de la memoria que no se vincularon directamente con los crímenes de la última dictadura militar. Posteriormente, en el transcurso del año 2014, la misma agencia del estado nacional señalizó la fosa común denominada "Cañadón del Muertos" en la localidad de Gobernador Gregores, contando en su inauguración además de con funcionarios provinciales y nacionales con el propio Osvaldo Bayer. Un año después, en el 2015, el Archivo Nacional de la Memoria (ANM) entregó a la Universidad Nacional de la Patagonia Austral y al Archivo Provincial de Santa Cruz, la versión digitalizada del "Diario de guerra" del coronel Varela. ${ }^{5}$ Asimismo el ANM elaboró un interesante material sobre las huelgas, donde se retoman gran parte de los argumentos de Bayer respecto a la interpretación del conflicto, que se encuentra a disposición de una descarga libre en su sitio web. Paralelamente a estas acciones el organismo nacional también propició el desarrollo de excavaciones en diversos sitios de la provincia con el fin de localizar los lugares de los fusilamientos y enterramientos. ${ }^{6}$ Al tiempo, acompañó la iniciativa provincial de patrimonialización de algunos espacios estrechamente relacionados con estos sucesos, como es el caso de la inauguración de algunos monumentos en la zona centro y norte de Santa Cruz.

En relación con las instituciones educativas, se sancionó la Ley Provincial № 3322 donde la obra de Osvaldo Bayer, Los vengadores de la Patagonia Trágica, sería de lectura obligatoria en los colegios secundarios así como la difusión de la película La Patagonia Rebelde. ${ }^{7}$ La legislación también menciona que los contenidos relacionados con las huelgas debían incluirse en los espacios curriculares de las asignaturas de Historia y Literatura del Nivel Secundario.

Esta incorporación de contenidos a las currículas escolares de las instituciones educativas santacruceñas, será declarada de interés por el Senado de la Nación, a partir de la iniciativa del entonces Senador por Santa Cruz, Pablo González. En el Proyecto de Declaración S-3033/13, del año 2013 se menciona: "Expresar su beneplácito por la incorporación en la planificación curricular de las áreas de Ciencias Sociales y de Literatura en los establecimientos educativos secundarios de la Provincia de Santa Cruz, el estudio y análisis de la obra literaria La Patagonia Rebelde del escritor Osvaldo Bayer, así como la exhibición del filme La Patagonia Trágica de Héctor Olivera dispuesto por la Ley Provincial 3322".

\footnotetext{
5 "El Archivo Nacional de la Memoria entregó 'Diario de Guerra' de Varela a la universidad". (https://www.unpa.edu.ar/noticia/el-archivo-nacional-de-la-memoria-entrego-diario-de-guerra-de-varela-launiversidad) (26/03/2015).

6 "Estancia Anita: Inician las excavaciones para encontrar restos de peones fusilados" (https://amanecerdeseadonoticias.com/2014/12/07/estancia-anita-inician-las-excavaciones-para-encontrar-restosde-peones-fusilados/) (12/07/2014).

7 Tiempo Sur Digital: "La "Patagonia Rebelde" de Bayer será lectura obligada en los colegios". (https://www.tiemposur.com.ar/nota/57217-la-patagonia-rebelde-de-bayer-sera-lectura-obligad) (21/7/2013).
} 
Dos años más tarde, por iniciativa de los Senadores por Santa Cruz, María E. Labado y Pablo González el Senado de la Nación distinguió con la mención de honor "Juana Azurduy de Padilla" al historiador y periodista Osvaldo Bayer, en mérito a su aporte a la cultura nacional y fundamentalmente por su obra más importante "La Patagonia Rebelde". 8

Es interesante destacar de este proceso de construcción por parte del estado provincial de una memoria oficial sobre las huelgas de los peones rurales, la centralidad que adquirió la figura del periodista y escritor Osvaldo Bayer en la vida pública de la provincia, charlas, conferencias, participación en actos públicos y hasta incluso se comprueba en la colocación de su nombre al colegio secundario $\mathrm{N}^{\circ} 41$ del popular Barrio San Benito de Río Gallegos, siendo la primera institución educativa del país que lleva el nombre del escritor.

\section{CONCLUSIONES}

El plan de trabajo de la beca de investigación se originó a partir del interés por reconstruir la presencia de textos literarios que permitan el abordaje de los hechos traumáticos en las escuelas. En particular, sobre la situación al respecto de las bibliotecas escolares de la ciudad de Río Gallegos. El contexto de pandemia que azotó al mundo no fue excepción en la localidad que también debió ajustarse a las indicaciones en torno a la emergencia sanitaria vinculada al COVID-19, que estableció el "Distanciamiento Social, Preventivo y Obligatorio" el cual restringió el desarrollo de las actividades escolares. En lo que respecta a la localidad de Río Gallegos incluso se ha extendido hasta la publicación de este informe.

La situación local condicionada por el contexto de pandemia limitó los alcances de la presente investigación en la cual tuvo que redefinirse nuevamente los aspectos a profundizar, es por ello que el abordaje que propusimos involucró indagar sobre la función de las bibliotecas escolares y las diferentes decisiones tomadas desde los gobiernos para el trabajo con la memoria colectiva en las escuelas. Por ello, este trabajo se conforma como una aproximación a la temática, un punto de partida para la profundización que se realizará a posterior con el trabajo dentro de las escuelas de la ciudad.

En este punto, y a partir del análisis realizado hemos podido dar cuenta a partir del análisis de un conjunto de políticas estatales que el pasado no es una dimensión pre-existente a las sociedades, muy por el contrario al interior de estas se desarrollan tensiones en torno a aquello que debe ser recordado y aquello que debe ser olvidado. En este sentido, la(s) memoria(s) son el resultado de conflictos y alianzas entre los discursos que compiten por una situación hegemónica.

Tanto en el caso de las huelgas obreras de 1921, como de la última dictadura militar, durante gran parte de la historia de la provincia de Santa Cruz, se configuraron mecanismos de olvido como forma de regulación del presente hacia aquel pasado. Con el advenimiento de la democracia y con mayor visibilidad durante las últimas gestiones del gobierno de la provincia de Santa Cruz, se impulsaron un conjunto políticas diversas que activaron evocaciones que plantearon un proceso inverso al tradicional olvido de las luchas obreras y de la dictadura militar.

${ }^{8}$ (https://www.tiemposur.com.ar/nota/87905-senado-de-la-naci\%F3n-homenaje\%F3-a-osvaldo-bayer) $(1 / 05 / 2015)$. 
El pasado de la provincia de Santa Cruz es un "pasado que no pasa", y que se manifiesta vigentes en las luchas por la memoria, en la imposibilidad del duelo. Las instituciones escolares, tan acostumbradas al tratamiento de pasados lejanos y cerrados, no está habituada a tratar temas que irrumpan frecuentemente en la escena pública, a pensar que el sentido sobre lo que pasó no esté fijado de manera definitiva. El pasado traumático, las huelgas obreras o la dictadura militar pocas veces aparecen como productos de una construcción histórica con avances, retrocesos, contradicciones.

Finalmente, y como señalamos en el trabajo, la construcción de una "pedagogía de la memoria" implica reflexionar sobre algunas cuestiones vinculadas a problemas que se desprenden de la propia tarea de la transmisión y que tienen que ver fundamentalmente con las preguntas por los contenidos y las formas en las que se enseña ese pasado y cómo hacerlo. En este punto, la reflexión sobre el lugar de la escuela y del conjunto de sus dispositivos para avanzar en ese camino resulta no sólo necesaria sino vital para la vida democrática.

\section{REFERENCIAS BIBLIOGRÁFICAS}

BOSSIÉ, F. (2009). De libros, bibliotecas y bibliotecarios en tiempos de dictadura [En línea]. 12. ${ }^{\circ}$ Congreso Internacional de Promoción de la Lectura y el Libro, 24 al 26 de abril de 2009, Ciudad Autónoma de Buenos Aires. Disponible en: http://www.fuentesmemoria.fahce.unlp.edu.ar/trab_eventos/ev.852/ev.852.pdf

CERUTTI, G. (2001). La historia de la memoria. Entre la fetichización y el duelo. En revista Puentes, 1 (3). Pp. 14-25.

GONZÁLEZ, M. (2014). Educación y memoria en Argentina. Documento de Trabajo $\mathrm{N}^{\circ} 207$. Instituto de Estudios Peruanos.

LEY NACIONAL 26.606 de 2006. Educación Nacional. 16 de Diciembre 2006. D. O. No. 31062 58ac89392ea4c.pdf

https://www.argentina.gob.ar/sites/default/files/ley-de-educ-nac-

LEY NACIONAL 26.917 de 2013. Nacional Sistema Nacional de Bibliotecas Escolares y Unidades de Información Educativas. 9 de Enero de 2014. http://www.bnm.me.gov.ar/giga1/normas/LE_26917.pdf

LEY NACIONAL 3305 de 2012. Educación Provincial. 22 de Noviembre 2012. https://dpegp.files.wordpress.com/2013/08/3305.pdf

LVOVICH, D. y BISQUERT, J (2008) La cambiante memoria de la dictadura. Discursos públicos, movimientos sociales y legitimidad democrática. Los Polvorines, Universidad Nacional de General Sarmiento, Biblioteca Nacional.

NORA, P. (2009). Los lugares de la memoria. Montevideo, Ediciones Trilce.

PÈTIT, M. (2011, Noviembre 10). Leer y hacer uso de una biblioteca escolar: ¿y eso, para qué sirve hoy en día? Bibliotecas escolares en tránsito, Santiago de Compostela. http://literacias.net/bibliodigital/index.php?page $=13 \& \mathrm{id}=64 \& \mathrm{db}=$

VALDIVIA, M. (2018, Septiembre 8). Bibliotecas, mediadores y memoria colectiva: tensiones y reflexiones en torno a las prácticas. http://humadoc.mdp.edu.ar:8080/xmlui/handle/123456789/666 